\title{
Multi-wavelength Switching Using Hybrid Plasmonic Add/drop Multiplexer
}

\author{
Surachai Tunsiri ${ }^{\mathrm{a},},{ }^{,}$Visrut Phetcharat ${ }^{\mathrm{b}}$, Nopparat Thammawongsa $^{\mathrm{c}}$ and Somsak Mitatha ${ }^{\mathrm{a}}$ \\ ${ }^{a}$ Hybrid Computing Research Laboratory, Faculty of Engineering, King Mongkut's Institute of Technology Ladkrabang, \\ Bangkok 10520,Thailand \\ ${ }^{\mathrm{b}}$ General Education Department, Udonthani Rajabhat University, Udonthani 41000, Thailand \\ ${ }^{c}$ Electronic Engineering Department, Faculty of Technology, Udonthani Rajabhat University, Udonthani 41000, \\ Thailand
}

*Corresponding Author: surachai.tu@kmitl.ac.th

\begin{abstract}
In this study, the novel device hybrid plasmonic add/drop multiplexer (HPAM) by surface plasmon polariton (SPPs) mechanism is demonstrated. Hybrids plasmonic add/drop multiplexer is used to optical multiwavelength switching networks make potential operator to generate and access of WDM/DWDM network. Methodology, the finite difference time domain (FDTD) techniques is applied by OptiFDTD programming which an experiment based on the characteristic of devices and schematic model design. Consequences, Au coupled provides a SPP mechanism operated on Add/drop ring multiplexer with multiwavelength switching. As a result, four wavelength users were selected $0.7,0.8,0.9$, and $1 \mathrm{um}$ with a various size of SPP ring Au coupled. The high Q factor $(28,500)$, $\mathrm{FSR}=1.4 \mathrm{THz}$ and FWHM at- $3 \mathrm{~dB}=10 \mathrm{~nm}$. A variety of applications, such as sensors, optical switching, and optical computation can be applied by plasmonic polariton mechanism.
\end{abstract}

Keywords: Hybrid plasmonic add/drop multiplexer, Multiwavelength switching, Surface plasmon polariton.

\section{Introduction}

The future networks with high transmission capacity, compactness by multilayer interconnection, and highly fast optical switching are greatly required. In order to enhance the capacity of an optical interconnect link as required, an effective solution is utilizing advanced multiplexing technologies such as wavelength division multiplexing (WDM), polarization division multiplexing (PDM), spatial division multiplexing (SDM), etc. Conventionally, the multiwavelength switchable function of the fiber laser is determined by the characteristics of the comb filter. Recently, multiwavelength fiber lasers have been widely applied in optical measurement, coherent pulse synthesis, optical spectroscopy, dense wavelength division multiplexed (DWDM) fiber communication systems, fiber optic sensors, and microwave photonics systems [1-4]. Especially, the use of multiwavelength photonic and multiwavelength erbium-doped fiber lasers (EDFL) has been investigated widely due to their advantages [5-7]. Narrower Multiwavelength pulse trains and mode locked multiple wavelengths can be potentially realized to gain the bandwidth [8]. A highly dual functions laser concentrated Erbium doped fiber with nonlinear polarization rotation (NPR) is studied. The EDFL-NPR operates as a 3 distinct wavelength lasers, evenly spaced at $1.7 \mathrm{~nm}$. [9] The key components on silicon-on-insulator ate realized the multiplexing technologies. Silicon photonics has provided a very attractive platform to build ultrasmall integrated photonic devices. [9] One need to improve is the fabrication processes to reduced the dispersion and scattering loss. In order to have a high $\mathrm{Q}$ factor, an optimal coupling ratio in proportion to the critical coupling condition [10]. An interesting to have an ultra-large free spectral range (FSR) is micro ring resonator filters with a large tenability range of wavelength 
Alternatively, waveguide ring resonators have been established for many applications [11-13], such as, tunable lasers, switching, modulators, compensators, and biosensors. The performance of ring resonators can support optical filter in Passive Optical Network (PON) applications but it is limited by internal losses. In this paper, the novel design of $\mathrm{ADD} / \mathrm{DROP}$ ring resonator with the mechanism of surface plasmon polariton (SPP). The proposed hybrids plasmonic add/drop multiplexer is used to generate and switch multiwavelength access to WDM/DWDM network. The finite difference time domain technique is applied by OptiFDTD programming which an experiment-based fabricated devices characteristic and schematic model design.

\section{Methodology}

Light propagates by total internal reflections at the waveguide core-cladding interface. A mode of wave may propagate through the fiber, formally, a mode can be analyzed by a wave equation derived from Maxwell's equations and subject to boundary conditions imposed by the optical waveguide depending on the relative size of the core compared to the considered wavelength. On the other hand, a proposals of plasmonic waveguides and integrated components have been change, e.g. by using metal stripes embedded in a dielectric $[14,15]$, SPP band gap structures [16], V-groves planar metal surface $[17,18]$ and hybrid ring resonator [19] which demonstrated a nonlinear mechanism to improve the optical waveguide.

The proposed hybrid plasmonic add-drop multiplexer made by InGaAsP and coupled with Au ring thin film. The time domain simulation by beam propagation model (BPM) is base on FDTD method. A proposed HPAM demonstrated in Fig. 1., which Through port fields and Drop port fields can be analyzed as in Eq.1 and 2

$$
\begin{aligned}
& E_{T h}=x_{1} y_{1} E_{i n}+\left(j x_{1} x_{2} y_{2} \sqrt{\kappa_{1}} E_{4} E_{L} E_{1}-x_{1} x_{2} \sqrt{\kappa_{1} \kappa_{2}} E_{R} E_{a d}\right) e^{M} \\
& E_{D r}=j x_{2} \sqrt{\kappa_{2}} E_{R} E_{L} e^{M}+x_{2} y_{2} E_{a d}
\end{aligned}
$$

where $\quad x_{1}=\sqrt{1-\kappa_{1}}, \quad y_{1}=\sqrt{1-\gamma_{1}}, \quad x_{2}=\sqrt{1-\kappa_{2}}, \quad y_{2}=\sqrt{1-\gamma_{2}}$, $M=-\frac{\alpha}{2} \frac{L}{2}-j k_{n} \frac{L}{2}, \kappa_{1}$ and $\kappa_{2}$ are the intensity coupling coefficient, $\gamma_{1}$ and $\gamma_{2}$ are the fractional coupler intensity loss, $\alpha$ is the attenuation coefficient, $\kappa_{n}=2 \pi / \lambda$ is the wave propagation number, $L=2 \pi R a d$, Rad is the radius of center ring resonator, $E_{L}$ and $E_{R}$ are circulated left and right ring light fields, respectively.

In the simulation model, gold $(\mathrm{Au})$ coupled for surface plasmon polariton mechanism obtained from Eq.3.

$$
\varepsilon(\omega)=\varepsilon_{\infty}-\frac{\omega_{p}}{\omega^{2}+i \gamma \omega}
$$

Which response by plasma frequency interaction was modeled by Drude [20] and 3D FDTD Perfectly matched layer by Berenger [21,22]. Using parameter of photonic and electrical model is shown in Table 1. The Au dielectric constant $\varepsilon_{\infty}$, the plasma frequency $\omega_{\mathrm{p}}$, and the collision frequency $\gamma$ at room temperature are $6.8890,8.9601 \times 10^{16}$ $\mathrm{s}^{-1}$, and $2.9715 \times 10^{13} \mathrm{~s}^{-1}$, respectively.

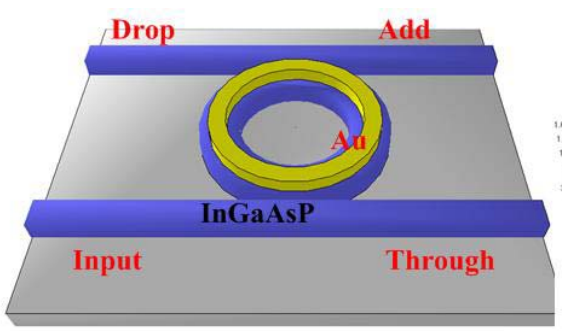

(a) Hybrid plasmonic add/drop multiplexer design

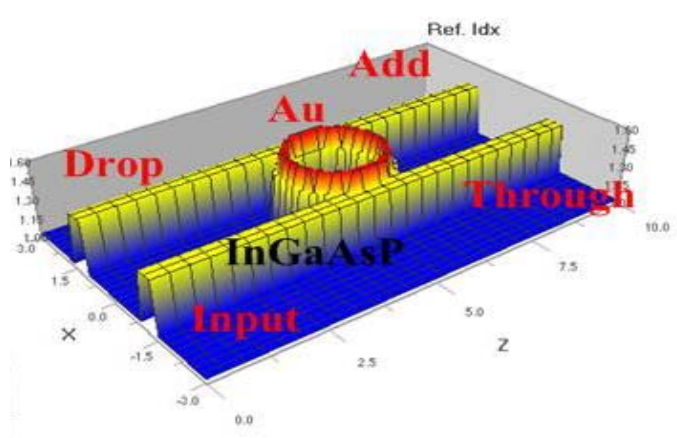

(b) Refractive index of HPAM

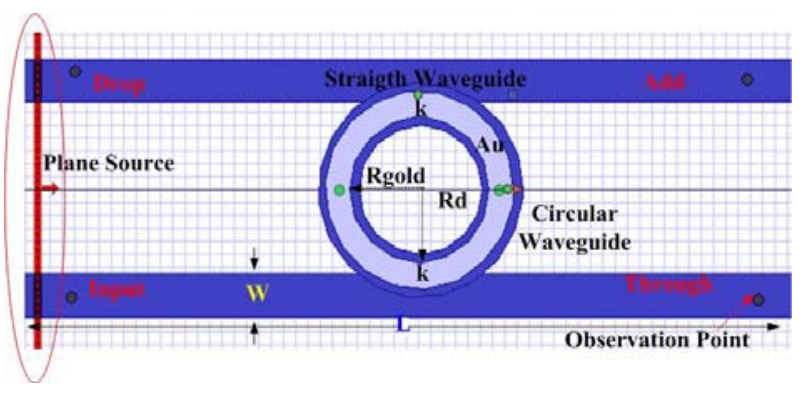

(c) Schematic model by OptiFDTD design

Fig. 1. Hybrid plasmonic add/drop multiplexer with varied Au coupled ring (a) HPAM model (b) Refractive index of HPAM and (c) Schematic model by OptiFDTD design 


\section{Simulation results}

Table 1. The parameter of photonic and electrical model for FDTD simulation

\begin{tabular}{|l|c|c|c|}
\hline \multicolumn{1}{|c|}{ Parameter } & Symbol & Typ. & Unit \\
\hline SiO2/InP substrate & Hsub & 2 & $\mu \mathrm{m}$ \\
\hline InGaAsP waveguide width & $\mathrm{W}$ & 400 & $\mathrm{~nm}$ \\
\hline InGaAsP waveguide height & $\mathrm{d}$ & 350 & $\mathrm{~nm}$ \\
\hline TE Propagation losses & $\mathrm{TE}$ & $2.5-4.5$ & $\mathrm{~dB} / \mathrm{cm}$ \\
\hline TM Propagation losses & $\mathrm{TM}$ & $2.5-4.5$ & $\mathrm{~dB} / \mathrm{cm}$ \\
\hline Gold film height & $\mathrm{HAu}$ & 100 & $\mathrm{~nm}$ \\
\hline Gold film width & WAu & 400 & $\mathrm{~nm}$ \\
\hline Propagation losses & $\alpha$ & 0.1 & $\mathrm{~dB} / \mu \mathrm{m}$ \\
\hline Propagation Length & Lsp & 10 & $\mu \mathrm{m}$ \\
\hline Mode effective index & Neff & 1.237 & \\
\hline
\end{tabular}

In OptiFDTD simulation, the HPAM can be resonated within center ring and the surface plasmon polariton mechanism was generated by $\mathrm{Au}$ rings, which depend on the used parameters and conditions in Table 1. The circularly signals can also be controlled to switch on the various wavelength by width of Au ring at the bottom, which can be useful to provide wavelength for a Remote Access Point (RAP) link control. The proposed results are shown in Fig.2., FDTD simulation for HPAM model (a) $\mathrm{W}=0.2 \mu \mathrm{m}$ which resonated at the $0.7 \mu \mathrm{m}$ wavelength, (b) $\mathrm{W}=0.3 \mu \mathrm{m}$ which resonated at the $0.8 \mu \mathrm{m}$ wavelength, (c) $\mathrm{W}=0.4 \mu \mathrm{m}$ which resonated at the $0.9 \mu \mathrm{m}$ wavelength and (d) $\mathrm{W}=0.5 \mu \mathrm{m}$ which resonated at the $1 \mu \mathrm{m}$ wavelength.
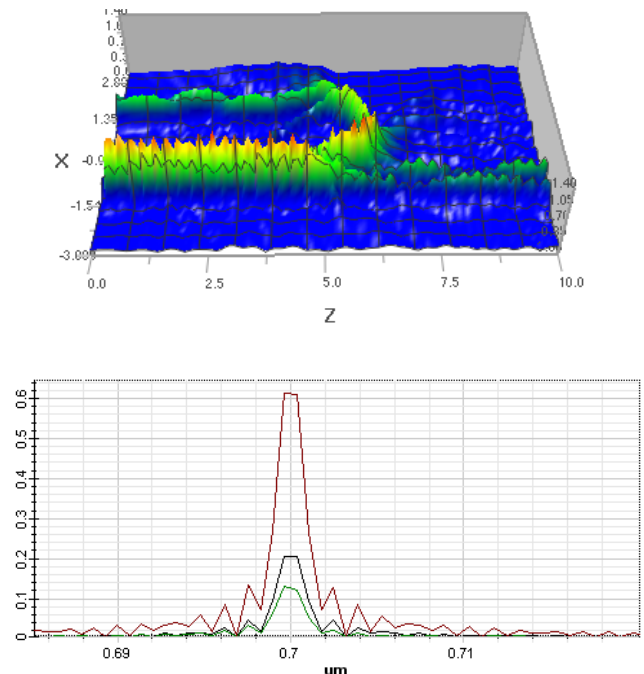

(a) $\mathrm{W}=0.2, \lambda=0.7 \mu \mathrm{m}$
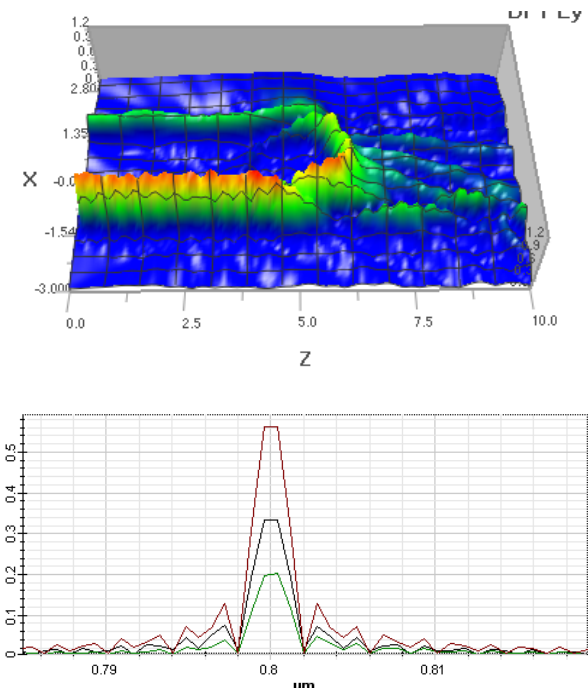

(b) $\mathrm{W}=0.3 \lambda=0.8 \mu \mathrm{m}$
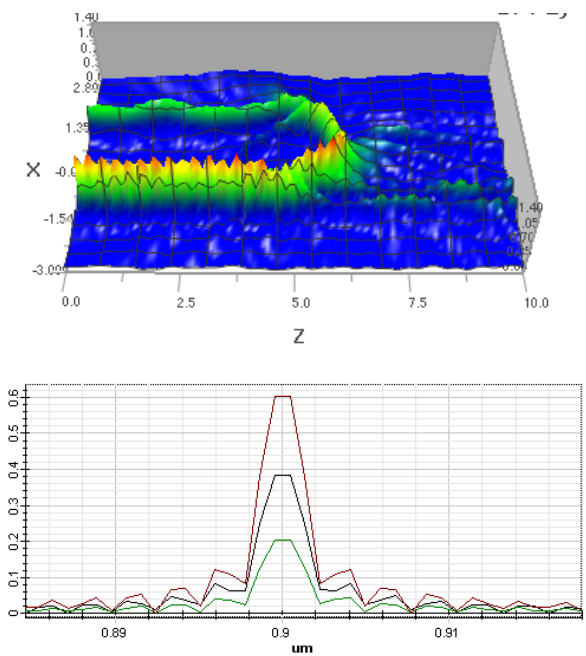

(c) $\mathrm{W}=0.4, \lambda=0.9 \mu \mathrm{m}$
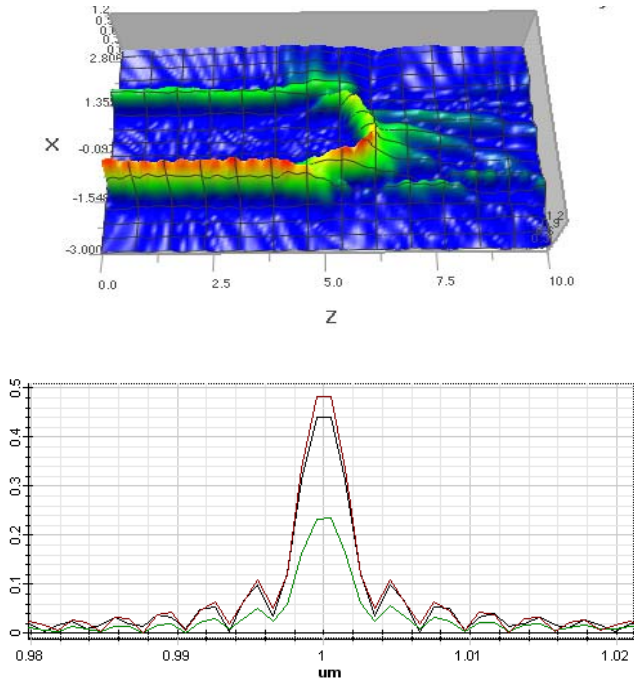

(d) $\mathrm{W}=0.5, \lambda=1 \mu \mathrm{m}$

Fig. 2. FDTD simulation for HPAM model (a) $\mathrm{W}=0.2 \mu \mathrm{m}$ resonated at the $0.7 \mu \mathrm{m}$ and

(b) $\mathrm{W}=0.3 \mu \mathrm{m}$ resonated at the $0.8 \mu \mathrm{m}$ and

(c) $\mathrm{W}=0.4 \mu \mathrm{m}$ resonated at the $0.9 \mu \mathrm{m}$ and

(d) $\mathrm{W}=0.5 \mu \mathrm{m}$ resonated at the $1 \mu \mathrm{m}$ wavelength 

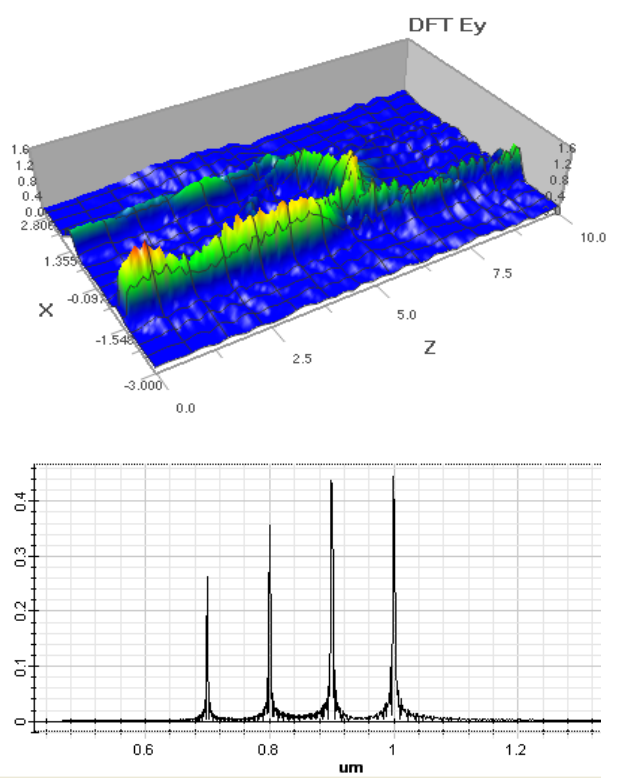

(a) Sub title.
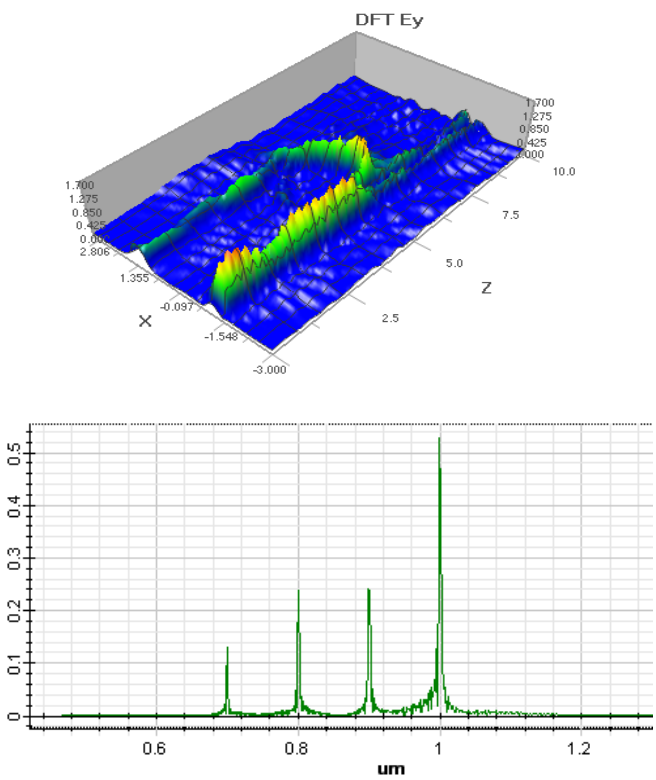

Fig. 3. FDTD simulation for HPAM model (a) 4 multiplex input signal and (b) 4 demultiplex output signal by OptiFDTD which only operate at $1 \mu \mathrm{m}$ wavelength

In Fig. 3, An 4 user Switched generated by HPAM ring at $1 \mu \mathrm{m}$ wavelength, a) 4 multiplex input signal and b) 4 demultiplex output signal by OptiFDTD which only operate at $1 \mu \mathrm{m}$ wavelength. As a result, we are obtained The high Q factor $(28,500)$ multiplexer with FSR $=1.4 \mathrm{THz}$ and FWHM at-3dB $=10 \mathrm{~nm}$. Therefore, the proposed HPAM design was used as a high passive switching, optical multiplexing network and many applications.

\section{Conclusions}

The design of hybrid plasmonic add/drop multiplexer (HPAM) by surface plasmon polariton (SPPs) mechanism is studied. Hybrids plasmonic add/drop multiplexer is used to optical multiwavelength switching networks make potential operator to generate and access of WDM/DWDM network. Methodology, the finite difference time domain (FDTD) techniques is applied by OptiFDTD programming which an experiment based on the characteristic of devices and schematic model design. Results, four wavelength users were selected $0.7,0.8,0.9$, and 1 um with a various size of $\mathrm{SPP}$ ring $\mathrm{Au}$ coupled. The high $\mathrm{Q}$ factor (28,500), $\mathrm{FSR}=1.4 \mathrm{THz}$ and $\mathrm{FWHM}$ at $-3 \mathrm{~dB}=10 \mathrm{~nm}$.

\section{References}

(1) P. Sharma, A. Kumar and V. K. Sharma : "Performance analysis of high speed optical network based on Dense Wavelength Division Multiplexing", Issues and Challenges in Intelligent Computing Techniques (ICICT), 2014 International Conference on Ghaziabad, pp. 446-449, 2014

(2) Z. N. Wang, Y. J. Rao, H. Wu, P. Y. Li, Y. Jiang, X. H. Jia, and W. L. Zhang : "Long-distance fiber-optic point sensing systems based on random fiber lasers", Opt. Express, Vol. 20, No. 16, pp. 17695-17700, 2012

(3) A. R. Sarmani, M. H. Abu Bakar, A. A. A. Bakar, F. R. M. Adikan, and M. A. Mahdi : "Spectral variations of the output spectrum in a random distributed feedback Raman fiber laser", Opt. Express, Vol. 19, No. 15, pp. 14152-14159, 2011

(4) Y. Zhang, F. Zhang and S. Pan : "A microwave photonic system with switchable functions based on cascaded polarization modulators", Optical Communications and Networks (ICOCN), 2014 13th International Conference on Suzhou, pp. 1-3, 2014

(5) 19. A. E. El-Taher, P. Harper, S. A. Babin, D. V. Churkin, E. V. Podivilov, J. D. Ania-Castanon, and S. K. Turitsyn : "Effect of Rayleigh-scattering distributed feedback on multiwavelength Raman fiber laser generation",Opt. Lett. Vol. 36, No. 2, pp.130-132, 2011

(6) A. M. R. Pinto, O. Frazão, J. L. Santos, and M. Lopez-Amo : "Multiwavelength fiber laser based on a photonic crystal fiber loop mirror with cooperative Rayleigh scattering", Appl. Phys. B, Vol. 99, No. 3, pp. 391-395, 2010 
(7) Z. Wang, H. Wu, M. Fan, Y. Li, Y. Gong, and Y. Rao : "Broadband flat-amplitude multiwavelength BrillouinRaman fiber laser with spectral reshaping by Rayleigh scattering", Opt. Express, Vol. 21, No. 24, pp. 29358-29363, 2013

(8) Y. Zhou, C. Zhang, P. C. Chui, and K. K. Y. Wong : "A tunable S-plus L-band continuous-wave single-longitudinal-mode fiber-optical parametric oscillator", IEEE Photon. Technol. Lett., Vol. 23, No. 20, pp. 1451-1453, 2011

(9) S. J. Tan, S. W. Harun : "Switchable pulse and multi-wavelength laser based on non-linear polarization rotation", International Conference on Technology, Informatics, Management, Engineering \& Environment (TIME-E2013), Bandung, Indonesia, June 23-26, 2013

(10) Hansuek Lee, Tong Chen, Jiang Li, Ki Youl Yang, Jeon Seokmin, Painter Oskar, Vahala Kerry J. : "Chemically etched ultrahigh-Q wedge-resonator on a silicon chip", Nature Photonics, Vol. 6, No. 6, pp. 369-373, 2012

(11)B.E. Little, S.T. Chu, H.A. Haus, and J. Foresi : "Microring resonator channel dropping filters", Journal of Lightwave Technology, Vol. 15, No. 6, pp. 998-1005, 1997

(12) Yadin Yoav and Orenstein Meir : "Parallel optical interconnects over multimode waveguides", J. Lightwave Technol. Vol. 24, No. 1, pp. 380-386, 2006

(13) Sanshui Xiao, Liu Liu, and Min Qiu : "Resonator channel drop filters in a plasmon-polaritons metal", Opt. Express, Vol. 14, No. 7, pp. 2932-2937, 2006

(14)P. Berini : "Plasmon-polariton waves guided by thin lossy metal films of finite width: Bound modes of symmetric structures", Phys. Rev. B, Vol. 61, No. 15, pp. 10484,2000

(15) T. Nikolajsen, K. Leosson, I. Salakhutdinov, and S. I. Bozhevolnyi : "Polymer-based surface plasmon polariton stripe waveguides at telecommunication wavelengths", Appl. Phys. Lett., Vol. 82, No. 5, pp. 668-670, 2003

(16) S. I. Bozhevolnyi, J. Erland, K. Leosson, P. M.W. Skovgaard, and J. M. Hvam : "Waveguiding in Surface Plasmon Polariton Band Gap Structures", Phys. Rev. Lett., Vol. 86, No. 14, pp. 3008-3011, 2001

(17) S. A. Maier, P. G. Kik, H. A. Atwater, S. Meltzer, E. Harel, B. E. Koel, and A. A. G. Requicha : "Local detection of electromagnetic energy transport below the diffraction limit in metal nanoparticle plasmon waveguides", Nat. Mat, Vol. 2, pp. 229-232, 2003
(18) S. I. Bozhevolnyi, V. S. Volkov, E. Devaux, and T. W. Ebbesen : "Channel plasmon-polariton guiding by subwavelength metal grooves", Phys. Rev. Lett, Vol. 95, No. 4, pp. 046802, 2005

(19) S. I. Bozhevolnyi, V. S. Volkov, E. Devaux, J.-Y. Laluet, and T. W. Ebbesen : "Channel plasmon subwavelength waveguide components including interferometers and ring resonators", Nature (London), Vol. 440, pp. 508-511, 2006

(20) Nopparat Thammawongsa,Khanthanou Luangxaysana, Saysamone Soysouvanh, and Somsak Mitatha : "The Simulation of Surface Plasmon Polariton based Photonic Integrated Devices by Modified Add-Drop", The 34th JSST Annual Conference: International Conference on Simulation Technology (JSST2015), At Toyama, Japan, Vol. 1, pp. 118-124, 2015

(21) K. Yee : "Numerical solution of initial boundary value problem involving Maxwell's equations in isotropic media”, IEEE Trans. Antennas and Propagation, Vol. 14, No. 3, pp. 302-307, 1966

(22) L.P. Berenger : "Perfectly matched layer for the FDTD solution of wave-structure interaction problem", IEEE Trans. Antennas Propag., Vol. 44, No. 1, pp.110-118, 1996 